\title{
Catecholamines in Alzheimer's Disease: A Systematic Review and Meta-Analysis
}

\author{
Xiongfeng Pan ${ }^{1}$, Atipatsa C. Kaminga ${ }^{1,2}$, Peng Jia ${ }^{3,4,5}$, Shi Wu Wen ${ }^{6,7}$, \\ Kwabena Acheampong ${ }^{1,8}$ and Aizhong Liu ${ }^{1 *}$
}

\begin{abstract}
${ }^{1}$ Department of Epidemiology and Health Statistics, Xiangya School of Public Health, Central South University, Changsha, China, ${ }^{2}$ Department of Mathematics and Statistics, Mzuzu University, Mzuzu, Malawi, ${ }^{3}$ Department of Land Surveying and Geo-Informatics, The Hong Kong Polytechnic University, Hong Kong, China, ${ }^{4}$ International Initiative on Spatial Lifecourse Epidemiology (ISLE), Hong Kong, China, ${ }^{5}$ Faculty of Geo-Information Science and Earth Observation, University of Twente, Enschede, Netherlands, ${ }^{6}$ Department of Obstetrics and Gynaecology, University of Ottawa, Ottawa, ON, Canada, ${ }^{7}$ Ottawa Hospital Research Institute, Ottawa, ON, Canada, ${ }^{8}$ Department of Public, School of Postgraduate Studies, Adventist University of Africa, Nairobi, Kenya
\end{abstract}

\section{OPEN ACCESS}

Edited by:

Huaibin Cai,

National Institutes of Health $(\mathrm{NIH})$

United States

Reviewed by:

Jingheng Zhou,

National Institute of Environmental Health Sciences (NIEHS),

United States

Lakshmi Rajagopal,

Northwestern University,

United States

*Correspondence:

Aizhong Liu

lazroy@live.cn

Received: 08 February 2020

Accepted: 26 May 2020

Published: 11 September 2020

Citation:

Pan X, Kaminga AC, Jia P, Wen SW,

Acheampong $K$ and Liu A (2020)

Catecholamines in Alzheimer's

Disease: A Systematic Review and

Meta-Analysis.

Front. Aging Neurosci. 12:184. doi: 10.3389/fnagi.2020.00184
Background and Purpose: Previous studies found inconsistent results regarding the relationship between Alzheimer's disease (AD) and catecholamines, such as dopamine $(\mathrm{DA})$, norepinephrine (NE), and epinephrine (EPI). Therefore, the purpose of this study was to perform a systematic review and meta-analysis to evaluate the results of previous studies on this relationship.

Method: Literature retrieval of eligible studies was performed in four databases (Web of Science, PubMed, Embase, and PsycARTICLES). Standardized mean differences (SMDs) were calculated to assess differences in catecholamine concentrations between the AD groups and controls.

Results: Thirteen studies met the eligibility criteria. Compared with the controls, significant lower concentrations of $\mathrm{NE}(\mathrm{SMD}=-1.10,95 \% \mathrm{Cl}:-2.01$ to $-0.18, p=$ $0.019)$ and $\mathrm{DA}(\mathrm{SMD}=-1.12,95 \% \mathrm{Cl}:-1.88$ to $-0.37, p=0.003)$ were observed in patients with $A D$. No difference was found in the concentrations of EPI between the two groups (SMD $=-0.74,95 \% \mathrm{Cl}:-1.85$ to $0.37, p=0.189$ ).

Conclusion: Overall, these findings are in line with the hypothesis that reduced NE and DA may be an important indicator for AD (Registration number CRD42018112816).

Keywords: catecholamines, dopamine, epinephrine, norepinephrine, Alzheimer's disease, meta-analysis

\section{INTRODUCTION}

Alzheimer's disease (AD) is characterized by attention deficits including apraxia, aphasia, agnosia, and progressive amnesia (Soldan et al., 2016). Its incidence has increased in recent years worldwide (Chibnik et al., 2017). Early screening focusing on AD biomarkers may not only be reliable and consistent but also help to implement timely interventions that could result in better prognosis and reduced disease burden (Fliessbach and Schneider, 2018).

Among the neurotransmitter abnormalities that have been investigated in $A D$, catecholamines, such as dopamine (DA), norepinephrine (NE), and epinephrine (EPI), have been intensively studied with regard to their roles in the neurotransmitter efflux that 
include mediating a number of cognition functions, working memory, recognition memory, and spatial memory (Yates et al., 1983; Liao et al., 2002; Nelson et al., 2011; Bensmann et al., 2019). Furthermore, recent neuropathological research found a link between molecular mechanisms, functional changes occurring in the catecholamines, and the neuropathology of $\mathrm{AD}$ (Youdim, 2018). Saldana et al. showed that dopaminergic cell death in the substantia nigra pars compacta ( $\mathrm{SNpc}$ ) was associated with the $\mathrm{AD}$ (Saldana et al., 2008). In addition, DA also plays a key role in both Amyloid beta formation and cognitive decline progression (Kumari et al., 2018).

Meanwhile, inconsistent results have been reported regarding the association of catecholamine concentrations with $\mathrm{AD}$ (Fitzgerald, 2010; Chalermpalanupap et al., 2013). However, some studies have demonstrated that DA concentrations were higher in AD patients than in controls (Yates et al., 1983). Existing data also suggested that NE and EPI concentrations may be elevated in some patients with AD (Fitzgerald, 2010). Although a systematic review on the pivotal role of DA and DA receptor in $\mathrm{AD}$ was published, subsequently there had been some similar studies that were published (Pan et al., 2019), suggesting that it was necessary to update the role of catecholamines in DA.

Furthermore, despite some qualitative literature reviews summarizing the association between the catecholamine concentrations and $\mathrm{AD}$, they were unable to quantitatively estimate the magnitude and significance of the association and address several critical methodological issues of the published studies concerning this theory (Bharath and Andersen, 2004). Thus, there was need to reassess this association and address the related critical methodological issues in the published studies using meta-analysis, which is widely known as the gold-standard method for data aggregation. To date, no such method has been employed to address these questions.

\section{METHODS}

\section{Protocol Registration}

This study used the Preferred Reporting Items for Systematic Review and Meta-Analysis (PRISMA) statement (Moher et al., 2009). Therefore, it was registered with the International Prospective Register of Systematic Reviews, PROSPERO, and the registration number is CRD42018112816.

\section{Search Strategy and Selection Criteria}

English articles published before October 1, 2019, were searched in the electronic databases, Web of Science, PubMed, Embase, and PsycARTICLES, to identify relevant studies on the relationship between catecholamine concentrations and AD. Search terms were designed with help from experienced librarians as follows: TI (Alzheimer's Disease OR Alzheimer Syndrome OR Alzheimer Dementia OR Alzheimer OR AD) AND TX (Catecholamine OR Catecholamines OR Dopamine OR Dopamin OR Epinephrine OR Norepinephrine OR Noradrenaline OR Noradrenalin OR Adrenaline OR Adrenalin). Hand searching in the reference lists of eligible studies was conducted in parallel by XP and AK, and any disagreement between them was resolved by the corresponding author (AL).

\section{Eligibility Criteria}

The eligibility criteria for inclusion of studies were specified as follows: (1) case-control studies or randomized controlled trials (RCTs), which investigated $\mathrm{AD}$ cases and healthy controls; (2) studies that used standardized diagnostic criteria for $A D$ based on the Diagnostic and Statistical Manual of Mental Disorders (DSM) or other international standardized criteria; and (3) studies that reported mean and standard deviation (SD) of catecholamines. On the contrary, studies were excluded if they (1) were case reports or review articles; (2) studied AD in combination with other mental disorders, or in vascular dementia patients, who used drugs that had influence on the catecholamine concentration medications or psychotropic; (3) reported results of non-humans or vitro experiments; and (4) were gray literature (i.e., unpublished reports).

\section{Data Extraction}

Data extraction was performed by two investigators (XP and $\mathrm{PJ})$ independently. Their disagreements were resolved through discussion with the corresponding author (AL). Information on the following variables was extracted from each eligible article: first author's surname, year published, geographical location, mean and standard deviation of age, gender distribution, method of $\mathrm{AD}$ assessment, and catecholamine measurements comprising type of sample, assay methods, sample storage temperatures, and mean and SD of the catecholamine concentrations studied. The data were organized and saved in Excel and EpiData 3.0.

\section{Quality Assessment}

The quality of the eligible studies was evaluated using the Newcastle-Ottawa Quality Assessment Scale (NOS) (Stang, 2010). This scale evaluates studies on three broad perspectives: (1) Selection, (2) Comparability, and (3) Outcome.

\section{Statistical Analysis}

The concentrations of the catecholamines were compared between the $\mathrm{AD}$ patients and controls by observing the significance of standardized mean difference (SMD) and its corresponding two-sided 95\% confidence interval (CI) (Higgins et al., 2003). The $\mathrm{R}$ software (version 3.5.1) was used to analyze these data. Heterogeneity between the eligible studies was quantified using the $I^{2}$ statistic, whose significance was evaluated using the Cochran's $Q$ statistic test. Specifically, maximal heterogeneity was indicated by $I^{2}=100 \%$, while no heterogeneity was indicated by $I^{2}=0 \%$ (Higgins et al., 2009). Also, publication bias was assessed by a funnel plot and Egger's test, when the number of eligible studies under consideration was at least 10 (Egger et al., 1997). Besides, sensitivity analysis was performed by redoing the meta-analysis each time an eligible study was removed from the analysis (Pan et al., 2018). Finally, all the hypothesis tests were two-sided with 5\% significance level.

\section{RESULTS}

\section{Literature Search}

A total of 707 articles were identified, 184 from Embase, 231 from Web of Science, 144 from PubMed, and 148 from 


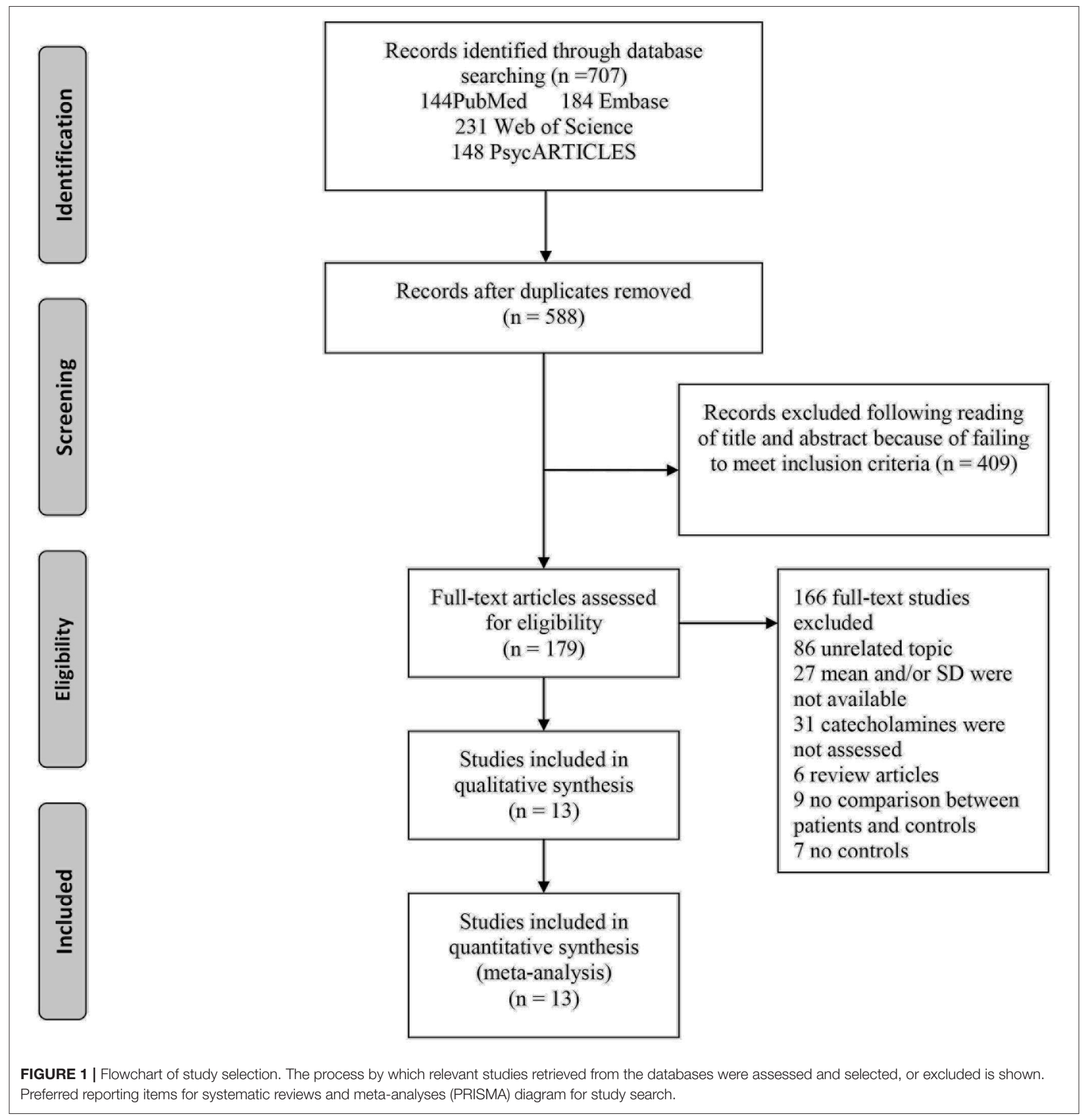

PsycARTICLES. Therefore, out of the 707 articles, 13 were considered as eligible for this study (Figure 1).

\section{Study Characteristics}

Table 1 presents the characteristics of the 13 eligible studies. Accordingly, EPI concentrations were compared between 167 $\mathrm{AD}$ participants and 98 controls, while the concentrations of NE were compared between 219 AD participants and 213 controls, and the concentrations of DA were compared between $212 \mathrm{AD}$ participants and 146 healthy controls.

\section{Overall Comparison}

Figure 2 shows the results of meta-analysis by the random-effects model. Thus, concentrations of EPI did not differ between the AD patients and healthy controls (SMD $=-0.74,95 \% \mathrm{CI}:-1.85$ to $0.37, p=0.189$ ) (Figure 2A). However, there were significantly lower concentrations of NE in the participants with $\mathrm{AD}$ than in 
TABLE 1 | Characteristics of studies included in meta-analysis of catecholamines in Alzheimer's disease.

\begin{tabular}{|c|c|c|c|c|c|c|c|c|c|}
\hline Study & Material & Country & NOS & Female & Mean age & AD assessment & Collection time & Methods & Frozen \\
\hline Bemelmans et al. (2007) & Plasma & Netherlands & 6 & $18(86 \%)$ & $85.2 \pm 5.1$ & DSM-IV & AM 9:00 & HPLC & $-20^{\circ} \mathrm{C}$ \\
\hline Elrod et al. (1997) & CSF & USA & 6 & $18(24 \%)$ & $69.0 \pm 6.0$ & DSM-IV & AM & $\mathrm{RIA}$ & $-70^{\circ} \mathrm{C}$ \\
\hline Kurup and Kurup (2003) & Plasma & India & 5 & $0(0 \%)$ & $67.5 \pm 5.7$ & ICD-10 & NR & HPLC & NR \\
\hline Lampe et al. (1989) & Plasma & USA & 8 & $0(0 \%)$ & $64.3 \pm 6.7$ & DSM-III & AM 9:00 & $\mathrm{RIA}$ & $-70^{\circ} \mathrm{C}$ \\
\hline Liu et al. (2011) & Urine & China & 5 & $0(0 \%)$ & $81.7 \pm 3.8$ & DSM-IV & NR & HPLC & $-20^{\circ} \mathrm{C}$ \\
\hline Peskind et al. (1995) & CSF & USA & 6 & $3(30 \%)$ & $69.5 \pm 2.5$ & DSM-III & AM 9:00 & HPLC & $-70^{\circ} \mathrm{C}$ \\
\hline Peskind et al. (1998) & CSF/Plasma & USA & 7 & $18(24 \%)$ & $69.0 \pm 6.0$ & DSM-III & AM 8:00 & $\mathrm{RIA}$ & $-70^{\circ} \mathrm{C}$ \\
\hline Umegaki et al. (2000) & Plasma & Japan & 7 & 66 (100\%) & $82.5 \pm 7.8$ & DSM-IV & AM 7:00 & HPLC & $-70^{\circ} \mathrm{C}$ \\
\hline Yates et al. (1983) & Brain & U.K. & 6 & $4(67 \%)$ & $72.0 \pm 18.0$ & Global dementia scale & NR & Other & $-70^{\circ} \mathrm{C}$ \\
\hline Adolfsson et al. (1979) & Brain & USA & 7 & $13(78 \%)$ & $81.3 \pm 7.2$ & Global dementia scale & $N R$ & Other & $-20^{\circ} \mathrm{C}$ \\
\hline Allard et al. (1990) & Brain & Sweden & 8 & $0(0 \%)$ & $82.0 \pm 6.0$ & DSM-III & NR & HPLC & $-70^{\circ} \mathrm{C}$ \\
\hline Dekker et al. (2018) & Brain & The Netherlands & 7 & $8(73 \%)$ & $81.3 \pm 7.6$ & DSM-IV & NR & HPLC & $-80^{\circ} \mathrm{C}$ \\
\hline Snowden et al. (2019) & Brain & U.K. & 7 & 7 (50\%) & $87.9 \pm 8.9$ & DSM-III & NR & LC-MS/MS & NR \\
\hline
\end{tabular}

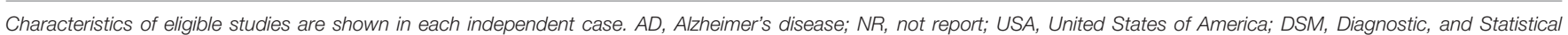

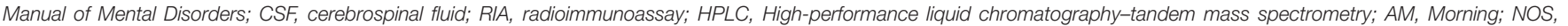

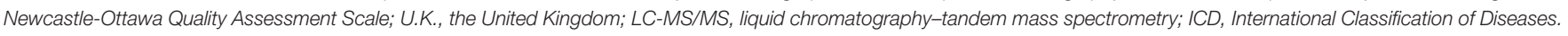

the healthy controls (SMD $=-1.10,95 \% \mathrm{CI}:-2.01$ to -0.18 , $p=0.019)$, and heterogeneity was considerable $\left(I^{2}=93.20 \%\right.$, Figure 2B). Regarding the eight studies that compared DA concentration levels between AD patients and healthy controls, significantly lower concentrations of DA were found in the AD patients than in the controls (SMD $=-1.12,95 \% \mathrm{CI}$ : -1.88 to $-0.37, p=0.003)$, but with considerable heterogeneity $\left(I^{2}=\right.$ $88.20 \%$, Figure 2C).

\section{Subgroup Analyses}

Table 2 shows the results of subgroup analyses. Although EPI concentrations did not generally differ between the AD patients and healthy controls, they were significantly higher in $\mathrm{AD}$ patients than in the controls for studies conducted in the United States (SMD $=0.42,95 \% \mathrm{CI}: 0.08$ to $0.76, p=0.014$ ), and with no heterogeneity detected $\left(I^{2}=0 \%\right)$. Similarly, when EPI was measured by the method of radioimmunoassay (RIA), there were significantly higher levels of EPI in patients with AD than in the healthy controls (SMD $=0.46,95 \% \mathrm{CI}: 0.08$ to 0.84 , $p=0.016)$.

In addition, lower NE concentrations were found in the $\mathrm{AD}$ participants than in the controls (SMD $=-2.73,95 \% \mathrm{CI}:-5.20$ to $-0.25, p=0.031$ ) with respect to the over 80 -year-old group, but these concentrations did not differ significantly between the two groups among the under the age of 80 group. Also, there were significantly lower concentrations of NE assayed by HPLC in the $\mathrm{AD}$ patients than in the controls (SMD $=-2.41,95 \% \mathrm{CI}$ : -3.97 to $-0.86, p=0.002$ ), but these concentrations did not differ significantly between the two groups with respect to the other assayed methods.

The results of sensitivity analysis indicated that there was no obvious influence of a single study on the outcomes of interest. Publication bias was not performed because the number of studies was $<10$ for each comparison.
Further, there were significant lower EPI concentrations in the male $\mathrm{AD}$ patients than in the healthy male controls $(\mathrm{SMD}=-5.24,95 \% \mathrm{CI}:-6.90$ to $-3.58, p<0.0001)$, but these concentrations did not differ significantly between the two groups among the female participants. Conversely, there were significant lower concentrations of NE in the female AD patients than in the healthy female controls $(\mathrm{SMD}=-7.05$, 95\% CI: -13.64 to $-0.46, p=0.036$ ), but these concentrations did not differ significantly between the two groups among the male participants. Similarly, the female AD patients had significantly lower DA concentrations than the healthy female controls (SMD $=-0.40,95 \% \mathrm{CI}:-0.70$ to $-0.10, p=0.009$ ), but these concentrations did not differ significantly between the two groups among the male participants.

\section{DISCUSSION}

Generally, this study found that DA and NE concentrations were lower in the $\mathrm{AD}$ patients than in the healthy controls, indicating that DA and NE may have an important role in the AD. However, the findings suggested that there was no significant association between EPI concentrations and AD, implying that more studies are needed to confirm this outcome. As the population ages worldwide, $\mathrm{AD}$ is increasingly becoming a serious problem and hence warrants the need to explore modifiable risk factors, which would help to find effective therapeutic interventions to reduce the prevalence of AD (Papp et al., 2017). Elderly people with lower DA and NE might require more intensive interventions to reduce risk of $\mathrm{AD}$. Thus, these findings are consistent with the catecholaminergic system dysfunction in the pathophysiology of $\mathrm{AD}$, which has been well documented. For example, some studies reported that dopaminergic system dysfunction could be detected in $\mathrm{AD}$ patients at early stages of the disease, even in the absence of $\mathrm{AD}$ signs, such as extrapyramidal signs (Albin et al., 2013, 2015). Also, according to the amyloid 


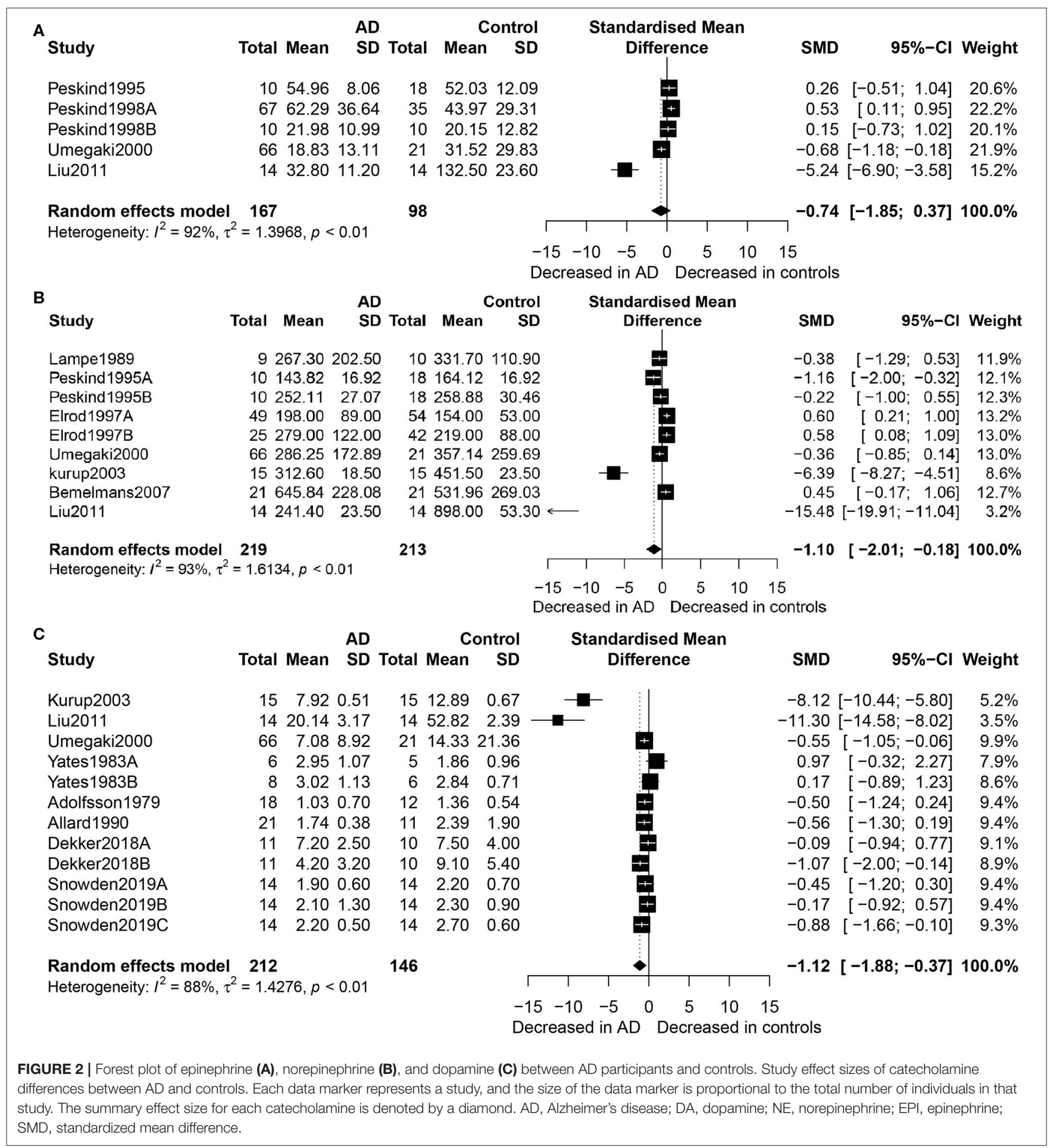

plaque hypothesis of $\mathrm{AD}$, Amyloid beta is neurotoxic and leads to the formation of neurofibrillary tangles and neuronal cell death in different regions of the brain, such as the cortical and hippocampus (Gordon et al., 2016). However, experimental studies have demonstrated that DA and its precursor L-dopa (L-3,4-dihydroxyphenylalanine) not only inhibited fibrillation but also appeared to dissolve existing Amyloid beta aggregates (Giunta et al., 2000).

As regards NE and EPI, previous studies indicated that they were associated with $\mathrm{AD}$. This association could be explained by the fact that the ventral tegmental area (VTA) dopaminergic neurons mainly project to the accumbens nucleus 
TABLE 2 | Subgroup analysis of dopamine, epinephrine, and norepinephrine between AD participants and controls.

\begin{tabular}{|c|c|c|c|c|c|c|c|c|}
\hline & Number of studies & SMD $(95 \% \mathrm{Cl})$ & $z$ & $P$-value & \multicolumn{4}{|c|}{ Heterogeneity } \\
\hline All & 5 & $-0.74[-1.85 ; 0.37]$ & -1.31 & 0.189 & 52.11 & $4<0.0001$ & 1.4 & $92.30 \%$ \\
\hline \multicolumn{9}{|c|}{ Study country } \\
\hline USA & 3 & $0.42[0.08 ; 0.76]$ & 2.45 & 0.014 & 0.80 & 20.6708 & 0.0 & $0.00 \%$ \\
\hline \multicolumn{9}{|l|}{ NOS } \\
\hline High & 3 & $-0.01[-0.85 ; 0.84]$ & -0.01 & 0.991 & 13.33 & 20.0013 & 0.5 & $85.00 \%$ \\
\hline Other & 2 & $-2.44[-7.83 ; 2.95]$ & -0.89 & 0.375 & 34.78 & $1<0.0001$ & 14.7 & $97.10 \%$ \\
\hline \multicolumn{9}{|c|}{ Assayed methods } \\
\hline Other & 3 & $-1.70[-3.74 ; 0.34]$ & -1.63 & 0.103 & 34.79 & $2<0.0001$ & 3.0 & $94.30 \%$ \\
\hline \multicolumn{9}{|l|}{ NE } \\
\hline All & 9 & $-1.10[-2.01 ;-0.18]$ & -2.36 & 0.019 & 117.68 & $8<0.0001$ & 1.6 & $93.20 \%$ \\
\hline \multicolumn{9}{|c|}{ Mean age } \\
\hline$>80$ & 3 & $-2.73[-5.20 ;-0.25]$ & -2.16 & 0.031 & 50.20 & $2<0.0001$ & 3.8 & $96.00 \%$ \\
\hline$\leq 80$ & 6 & $-0.84[-1.89 ; 0.21]$ & -1.57 & 0.116 & 66.08 & $5<0.0001$ & 1.5 & $92.40 \%$ \\
\hline \multicolumn{9}{|c|}{ Assayed methods } \\
\hline HPLC & 6 & $-2.41[-3.97 ;-0.86]$ & -3.04 & 0.002 & 94.03 & $5<0.0001$ & 3.2 & $94.70 \%$ \\
\hline Other & 3 & $0.41[-0.04 ; 0.87]$ & 1.80 & 0.073 & 3.97 & 20.1373 & 0.1 & $49.60 \%$ \\
\hline \multicolumn{9}{|l|}{ Gender } \\
\hline Female & 3 & $-7.05[-13.64 ;-0.46]$ & -2.10 & 0.036 & 68.60 & $2<0.0001$ & 31.94 & $97.10 \%$ \\
\hline Female & 9 & $-0.40[-0.70 ;-0.10]$ & -2.61 & 0.009 & 10.23 & 80.2489 & 0.0 & $21.80 \%$ \\
\hline Male & 3 & $-6.53[-13.47 ; 0.40]$ & -1.85 & 0.065 & 71.53 & $2<0.0001$ & 36.2 & $97.20 \%$ \\
\hline \multicolumn{9}{|c|}{ Assayed methods } \\
\hline HPLC & 6 & $-2.77[-4.36 ;-1.18]$ & -3.41 & 0.001 & 82.06 & $5<0.0001$ & 3.3 & $93.90 \%$ \\
\hline Other & 6 & $-0.28[-0.70 ; 0.13]$ & -1.34 & 0.181 & 7.14 & $5 \quad 0.2104$ & 0.1 & $30.00 \%$ \\
\hline
\end{tabular}

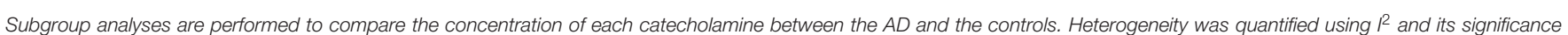

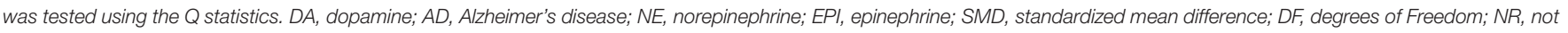

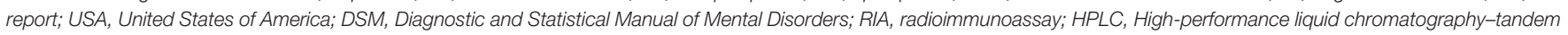
mass spectrometry; AM, Morning; NOS, Newcastle-Ottawa Quality Assessment Scale; U.K., the United Kingdom.

(Nacc), amygdala, and the cerebral cortex, while the substantia nigra pars compacta neurons (SNpc) mainly project to the dorsal striatum (Figure 3A) (Krashia et al., 2017). Thus, several studies have suggested that tau lesions in the primary source of subcortical NE and EPI, and locus coeruleus (LC), may be the identifiable pathogenesis of AD (Zarow et al., 2003). Further, LC cells secrete the neurotransmitter NE in the brain, and the NE secreted by LC has important effects on the following regions of the brain: motor activity (cerebellum), spatial attention (prefrontal and parietal cortices, thalamus, and amygdala), learning and memory (hippocampus, prefrontal cortex, and amygdala), and arousal (cerebral cortex, thalamus, and hypothalamus; Figure 3A; Zarow et al., 2003). Moreover, several studies reported that tyrosine increases the prefrontal cortex dopamine and norepinephrine levels (Jaskiw et al., 2006). Considering oxidative stress, reduced concentrations of $\mathrm{NE}$ and IP3 contribute to the endoplasmic reticulum stress and impairment of proteins processing (Andres-Benito et al., 2017; 
Anton-Fernandez et al., 2017). PKA hyper-phosphorylates Tau protein, which leads to microtubule disaggregation and the formation of neurofibrillary tangles (NFTs) (Figure 3B; Kennedy et al., 2004).

In addition, other studies indicated that rotigotine, a DA receptor agonist, induces changes in both cortical excitability (increased) and central cholinergic transmission (restored) in AD patients (Martorana et al., 2013). Furthermore, the results of recent basic research supported the hypothesis that reduction of $\mathrm{NE}$ is associated with the pathophysiology of $\mathrm{AD}$ (Vermeiren et al., 2016). In particular, it was noted that cognitive impairment coincided with the LC atrophy, LC degeneration, and NE loss, and these have been considered as early stage signs of $\mathrm{AD}$ pathogenesis (Daulatzai, 2016). Furthermore, there is growing evidence suggesting that, while the physical process of LC neuron degeneration exacerbates $\mathrm{AD}$-like neuropathology, the reduced NE impairs synaptic plasticity and cognitive performance (Rajkumar et al., 2017). Moreover, the inflammatory response induced and augmented by reduction of NE has been a key mechanism contributing to the initiation and progression of $\mathrm{AD}$ pathogenesis, microglia, endothelia, and astrocytes, which are among the major targets of inflammatory response (Bardou et al., 2014; Choi et al., 2015).

In general, $\mathrm{NE}$ is an anti-inflammatory molecule, and the deleterious effect of NE degeneration on the neuro-inflammatory response is the dysfunction of cellular machinery involved in Amyloid beta plaque metabolism and clearance (Lodeiro et al., 2017). Also in some animal studies, atomoxetine, a norepinephrine transporter inhibitor, demonstrated that treatment of 5XFAD transgenic mice (with Amyloid beta burden) elevated brain NE concentrations, increased expression of Amyloid beta clearance enzymes, reduced inflammatory changes, and improved spatial memory (Kalinin et al., 2012). Other clinical studies also suggested that atomoxetine was associated with improvement of cognition (Griffiths et al., 2018).

Although EPI was not associated with AD generally, there could be other moderators involving EPI that could be associated with $\mathrm{AD}$. Therefore, the EPI concentrations in $\mathrm{AD}$ were considered for subgroup analyses just as the concentrations of DA and NE in AD (Dwibedi et al., 2018). Thus, according to the results of subgroup analysis, the association between $\mathrm{EPI}$ and $\mathrm{AD}$ was significant for studies conducted in the USA, unlike for studies conducted in the other countries. Nonetheless, EPI and NE results were inconsistent, probably because compensatory mechanisms may produce increased release of these neurotransmitters after loss of LC cells in $\mathrm{AD}$ (Fitzgerald, 2010).

However, it should also be noted that outlier values existed in this meta-analysis. For example, the forest map shows that the studies by Liu et al. and Kurup et al. may be outliers. There are several possible reasons for this. Firstly, these studies were conducted in China and India, respectively, whereas most of the other studies were conducted in Europe or America; hence, there may be differences due to population characteristics and ethnicity. Secondly, the quality score of each suspected outlier was lower ( 5 points) than that of the other studies in this meta-analysis.
Similarly, significantly higher concentrations of EPI were found in the AD patients than in the controls when EPI was assayed by RIA, whereas no significant differences in the same were observed between patients with $\mathrm{AD}$ and controls when it was assayed by other methods. These results may suggest that RIA could be a more reliable assay method for EPI compared with other technologies, when assessing the relationship between EPI concentrations and AD (Raum and Swerdloff, 1981).

Also, subgroup analysis indicated that NE concentrations were lower in $\mathrm{AD}$ patients than in the controls for subjects aged at least 80 . Previous studies proposed that age-associated $\mathrm{NE}$ deficiency is implicated in the pathophysiology of AD. For instance, some animal studies demonstrated that NE can treat and relieve the oxidative stress of aging rats cells (Schraml et al., 2007). Moreover, there was a correlation between a decrease in beta-adrenergic receptor-mediated neuromodulatory actions and NE, which contributed to the age-related memory declines (Bickford, 1995). Some clinical studies also suggested that agingrelated emotional memory deficits could be reversed by $\mathrm{NE}$ via regulating the stability of surface AMPA receptors (Luo et al., 2015). These findings, therefore, are consistent with the association between $\mathrm{NE}$ and $\mathrm{AD}$ found in this study among subjects with at least 80 years of age, implying that NE may play a pivotal role in the pathophysiology of $\mathrm{AD}$; hence, it may be a potential candidate for treating aging-related memory deficits.

To date, a number of factors have been proposed to cause age-induced damage to the brain, including oxidative stress, free radical damage, and intracellular fibrillary tangles, which can be modified by aging, and have been associated with occurrence of AD (Mohsenzadegan and Mirshafiey, 2012; Tatsumi et al., 2014). Moreover, DA deficiency was found to contribute to age-related changes (Aliev et al., 2014). Meanwhile, previous research showed that DA density progressively reduced with aging and more severely in cases with AD (Rieckmann et al., 2018). Therefore, future clinical trials are needed to verify the potential therapeutic effectiveness of dopaminergic drugs in $\mathrm{AD}$, particularly for $\mathrm{AD}$ patients aged 80 years or older.

Furthermore, the important neurotransmitters, catecholamines, are produced mainly in the sympathetic nervous system, adrenal medulla, and the brain (Ounissi et al., 2015). Therefore, after catecholamine synthesis, they are conserved in postganglionic neurons in membrane-bound storage vesicles within the chromaticity globules of the adrenal medulla and released into the circulation (Ounissi et al., 2015). Brain tissue or cerebrospinal fluid (CSF) catecholamine samples have a high degree of correlation with brain aging and progression of $\mathrm{AD}$ (Tank and Lee, 2015; Zhang and Gong, 2016). However, these two samples were difficult to obtain, so plasma was selected for most of the studies. Thus, previous studies have shown that plasma catecholamine analysis technology and stability have been extensively studied (Peaston and Weinkove, 2004). Detailed steps of collection and treatment of plasma catecholamines are described in the previous studies. For example, centrifugal heparinized blood is sufficient within $30 \mathrm{~min}$ of collection. Once plasma is isolated from blood cells, catecholamines can be stored for up to 1 year at $-70^{\circ} \mathrm{C}$ (Peaston and Weinkove, 2004). However, plasma catecholamine samples are affected 

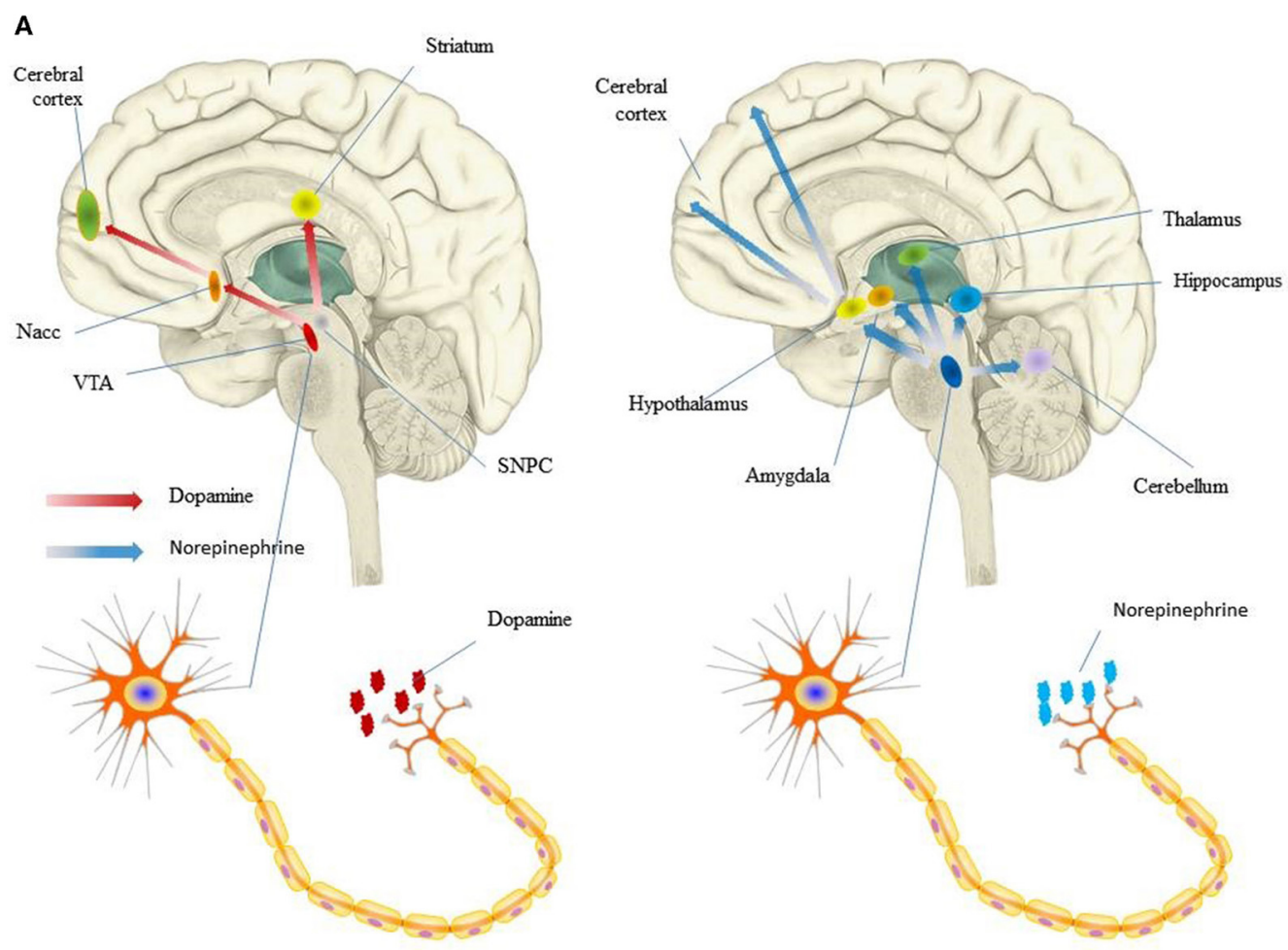

B

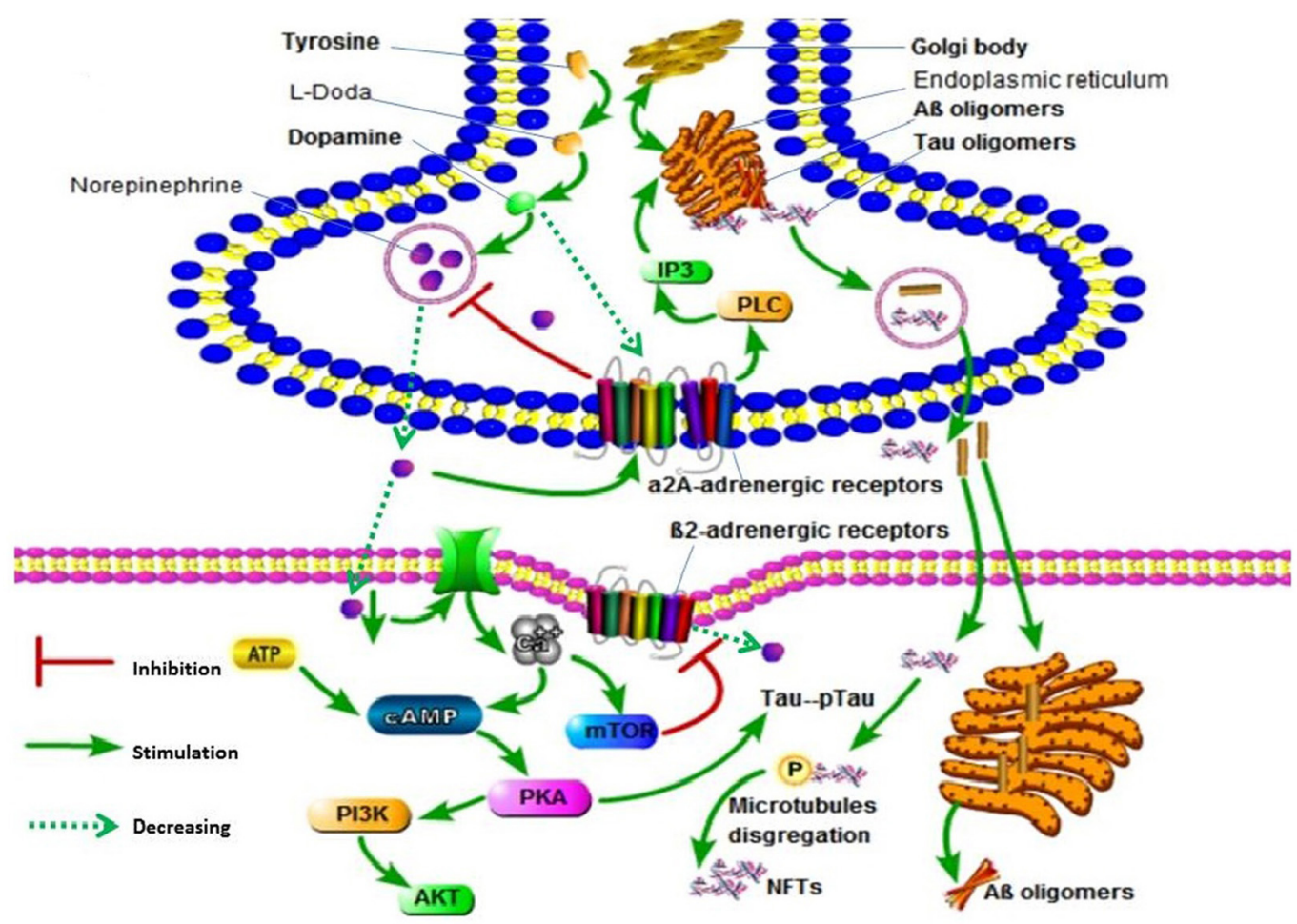

FIGURE 3 | Dopamine and norepinephrine flow in human brain and its molecular events in synaptic. (A) Digital illustration of direction of dopamine and norepinephrine flow in human brain. Ventral tegmental area, VTA; accumbens nucleus, Nacc; Substantia nigra pars compacta neurons, SNpc. (B) Molecular events occurring in Alzheimer's disease following locus coeruleus dopamine and norepinephrine loss and autophagy impairment. pTau, PKA hyper-phosphorylates Tau protein; Neurofibrillary tangles, NFTs. In (A), the brain and nerve cells are derived from the network, without copyright. In (B), all the pictures are hand-drawn by us without any copyright. In this figure is drawn by AK. 
by diurnal variation, although 24 -h urinary catecholamines circumvent this variation. Noteworthy, the stability of 24-h urinary catecholamines is still controversial; for example, catecholamine urine samples are prone to auto-oxidation at an alkaline pH (Grouzmann and Lamine, 2013). Overall, further research is needed to alert laboratory technicians of the possible pre-analysis problems of differences between different samples, including the standardization of the procedures for sample collection, transport, and storage conditions.

Additionally, contrary to EPI concentrations, significantly lower NE concentrations were found in $\mathrm{AD}$ patients than in the controls, when NE samples were assayed by HPLC, but no such difference was found between the two groups, when NE samples were assayed by other methods. Thus, compared with other assay technologies, this result may suggest that HPLC may be more reliable when investigating the relationship between $\mathrm{NE}$ concentrations and AD (Hows et al., 2004).

Furthermore, subgroup analysis indicated that females with $\mathrm{AD}$ had lower concentrations of $\mathrm{NE}$ than their counterparts, female controls. However, no such significant difference was apparent between the males with $\mathrm{AD}$ and male controls. It is well-known that females are at a greater risk of developing AD (Ferretti et al., 2018). Although the sex-specific clinicopathological AD phenotypes are largely unexplored, multiple factors have been suggested to underlie the observed association between $\mathrm{AD}$ and gender (Mosconi et al., 2017; Ferretti et al., 2018). Several lines of evidence support the gender-mediated genetic and endocrine hypothesis of AD. For example, multimodality brain imaging indicated sex differences in the development of $\mathrm{AD}$. Also, it indicated that endocrine (progesterone and estrogen) changes play an important role in the process of female preclinical AD phase, which occurs during the female early aging process and coincides with the endocrine transition of perimenopause (Mosconi et al., 2017). Moreover, experimental studies demonstrated that NE neurons within the nucleus tractus solitarii and ventrolateral medulla represent gonadal steroid-dependent components of several neural networks regulating estrogen and progesterone (Haywood et al., 1999). In particular, estrogen modulated by $\mathrm{NE}$ can prevent memory impairment in AD (Martinez-Morales et al., 2001). Other clinical studies also observed low plasma NE concentrations in the elderly female with AD (Umegaki et al., 2000). Therefore, these previous findings are consistent with the results of this study that NE concentrations differed between the $\mathrm{AD}$ patients and controls with respect to the female gender. Conversely, male $\mathrm{AD}$ patients showed a significant decrease in EPI concentrations when compared with male controls, but these concentrations did not significantly differ between females with $\mathrm{AD}$ and female controls. Nevertheless, considering the small sample size of the male group, this result may not be reliable. Therefore, more studies are needed in the future to explore the role of EPI concentrations in the pathophysiological process of $\mathrm{AD}$ in males and females. Additionally, with respect to DA, subgroup analyses indicated that females with $\mathrm{AD}$ had lower concentrations of DA than their counterparts, female controls. In spite of that, no such significant difference was apparent between males with $\mathrm{AD}$ and male controls. Current studies of individuals with $\mathrm{AD}$ provide evidence of alterations in the neuroendocrine system that dopamine and acetylcholine are affected by sex steroid hormones (Giacobini and Pepeu, 2018). One explanation could be that sex hormones exert trophic effects on the cholinergic system, while acetylcholine is involved in dopaminergic mediators, which are responsible for progressive synaptic disarrangement, impairment of neurotransmission, and cell loss. Thus, this plays a key role in brain damage and aging and may explain why females are more vulnerable than males to the development of $\mathrm{AD}$ symptoms, which aggravate after perimenopause. Therefore, addressing gender differences in $\mathrm{AD}$ would be crucial for the development of precise and effective therapeutics for $\mathrm{AD}$. In summary, gender-related differences in neural anatomy and function are starting to emerge, and gender might constitute an important factor for $\mathrm{AD}$ patients' stratification and personalized treatment.

There are some limitations to consider when interpreting the results of this review. First, the number of included participants in the eligible studies was relatively small. Next, all the eligible studies for this meta-analysis were cross-sectional studies; therefore, causal relationship or association could not be established. Last, the present study did not consider confounders, which may affect concentrations of catecholamines, such as blood pressure, smoking, body mass index, alcohol drinking status, and physical activity (Walker and Kane, 2002; Saxena et al., 2014). That is, the majority of the eligible studies for this meta-analysis did not report these confounders. Therefore, it is necessary that future studies on this subject should take the preceding confounders into consideration.

\section{CONCLUSION}

This meta-analysis suggests that $\mathrm{AD}$ was associated with decreased concentrations of DA and NE, but not with the changes in EPI concentrations. Although the molecular underpinnings of the decrease of $\mathrm{DA}$ and $\mathrm{NE}$ in $\mathrm{AD}$ remain to be confirmed, the foregoing results might open up new perspectives in the early diagnosis, identification of novel neuroimaging biomarkers, and provision of novel targets for pharmacological interventions. Nevertheless, further research is needed to ascertain whether NE could be used as a diagnostic tool.

\section{DATA AVAILABILITY STATEMENT}

All datasets generated for this study are included in the article/Supplementary Material.

\section{AUTHOR CONTRIBUTIONS}

$\mathrm{AL}$ and XP participated in study design. XP, PJ, KA, and AK contributed to the analysis, quality assessment data collection, statistical analyses and interpretation of results, and drafting and revising the manuscript. SW participated in the critical revision discussion and manuscript revision. All authors contributed to the article and approved the submitted version. 


\section{ACKNOWLEDGMENTS}

This study was funded by Canadian Institutes of Health Research (CIHR grant \# FDN-148438) and the authors would like to thank Central South University Library for the literature search help.

\section{REFERENCES}

Adolfsson, R., Gottfries, C. G., Roos, B. E., and Winblad, B. (1979). Changes in the brain catecholamines in patients with dementia of Alzheimer type. British J. Psychiatry 135, 216-223. doi: 10.1192/bjp.135.3.216

Albin, R. L., Burke, J. F., Koeppe, R. A., Giordani, B., Gilman, S., and Frey, K. A. (2013). Assessing mild cognitive impairment with amyloid and dopamine terminal molecular imaging. J. Nucl. Med. 54, 887-893. doi: 10.2967/jnumed.112.112599

Albin, R. L., Fisher-Hubbard, A., Shanmugasundaram, K., Koeppe, R. A., Burke, J. F., and Camelo-Piragua, S. (2015). Post-Mortem evaluation of amyloiddopamine terminal positron emission tomography dementia classifications. Ann. Neurol. 78, 824-830. doi: 10.1002/ana.24481

Aliev, G., Shahida, K., Gan, S. H., Firoz, C., Khan, A., and Abuzenadah, A. M. (2014). Alzheimer disease and type 2 diabetes mellitus: the link to tyrosine hydroxylase and probable nutritional strategies. CNS Neurol. Disord Drug Targets 13, 467-477. doi: 10.2174/18715273113126660153

Allard, P., Alafuzoff, I., Carlsson, A., Eriksson, K., Ericson, E., and Gottfries, C. G. (1990). Loss of dopamine uptake sites labeled with [3H]GBR-12935 in Alzheimer's disease. Euro. Neurol. 30, 181-185. doi: 10.1159/000117341

Andres-Benito, P., Fernandez-Duenas, V., Carmona, M., Escobar, L. A., TorrejonEscribano, B., and Aso, E. (2017). Locus coeruleus at asymptomatic early and middle Braak stages of neurofibrillary tangle pathology. Neuropathol. Appl. Neurobiol. 43, 373-392. doi: 10.1111/nan.12386

Anton-Fernandez, A., Aparicio-Torres, G., Tapia, S., DeFelipe, J., and Munoz, A. (2017). Morphometric alterations of Golgi apparatus in Alzheimer's disease are related to tau hyperphosphorylation. Neurobiol. Dis. 97, 11-23. doi: 10.1016/j.nbd.2016.10.005

Bardou, I., Kaercher, R. M., Brothers, H. M., Hopp, S. C., Royer, S., and Wenk, G. L. (2014). Age and duration of inflammatory environment differentially affect the neuroimmune response and catecholaminergic neurons in the midbrain and brainstem. Neurobiol. Aging 35, 1065-1073. doi: 10.1016/j.neurobiolaging.2013.11.006

Bemelmans, K. J., Noort, A., De Rijk, R., Middelkoop, H. A. M., Van Kempen, G. M. J., and Goekoop, J. G. (2007). Plasma cortisol and norepinephrine in Alzheimer's disease: opposite relations with recall performance and stage of progression. Acta Neuropsychiatrica 19, 231-237. doi: 10.1111/j.1601-5215.2006.00172.x

Bensmann, W., Zink, N., Roessner, V., Stock, A. K., and Beste, C. (2019). Catecholaminergic effects on inhibitory control depend on the interplay of prior task experience and working memory demands. J. Psychopharmacol. 33, 678-687. doi: 10.1177/0269881119827815

Bharath, S., and Andersen, J. K. (2004). Catecholamines and protein deposition in Parkinson's and Alzheimer's disease: old medicine, new targets. Rejuvenation Res. 7, 92-94. doi: 10.1089/1549168041553071

Bickford, P. (1995). Aging and motor learning: a possible role for norepinephrine in cerebellar plasticity. Rev. Neurosci. 6, 35-46. doi: 10.1515/REVNEURO.1995.6.1.35

Chalermpalanupap, T., Kinkead, B., Hu, W. T., Kummer, M. P., Hammerschmidt, T., and Heneka, M. T. (2013). Targeting norepinephrine in mild cognitive impairment and Alzheimer's disease. Alzheimers Res. Ther. 5:21. doi: $10.1186 /$ alzrt175

Chibnik, L. B., Wolters, F. J., Backman, K., Beiser, A., Berr, C., and Bis, J. C. (2017). Trends in the incidence of dementia: design and methods in the Alzheimer cohorts consortium. Eur. J. Epidemiol. 32, 931-938. doi: $10.1007 /$ s10654-017-0320-5

Choi, H. S., Park, J. H., Ahn, J. H., Hong, S., Cho, J. H., and Won, M. H. (2015). The anti-inflammatory activity of duloxetine, a serotonin/norepinephrine reuptake

\section{SUPPLEMENTARY MATERIAL}

The Supplementary Material for this article can be found online at: https://www.frontiersin.org/articles/10.3389/fnagi. 2020.00184/full\#supplementary-material

inhibitor, prevents kainic acid-induced hippocampal neuronal death in mice. $J$. Neurol. Sci. 358, 390-397. doi: 10.1016/j.jns.2015.10.001

Daulatzai, M. A. (2016). Dysfunctional sensory modalities, locus coeruleus, and basal forebrain: early determinants that promote neuropathogenesis of cognitive and memory decline and Alzheimer's disease. Neurotox Res. 30, 295-337. doi: 10.1007/s12640-016-9643-3

Dekker, A. D., Vermeiren, Y., Carmona-Iragui, M., Benejam, B., Videla, L., and Gelpi, E. (2018). Monoaminergic impairment in down syndrome with Alzheimer's disease compared to early-onset Alzheimer's disease. Alzheimers Dement (Amst) 10, 99-111. doi: 10.1016/j.dadm.2017.11.001

Dwibedi, N., Findley, P. A., Wiener, R. C., Shen, C., and Sambamoorthi, U. (2018). Alzheimer disease and related disorders and out-of-pocket health care spending and burden among elderly medicare beneficiaries. Med. Care 56, 240-246. doi: 10.1097/MLR.0000000000000869

Egger, M., Davey, S. G., Schneider, M., and Minder, C. (1997). Bias in meta-analysis detected by a simple, graphical test. BMJ 315, 629-634. doi: 10.1136/bmj.315.7109.629

Elrod, R., Peskind, E. R., DiGiacomo, L., Brodkin, K. I., Veith, R. C., and Raskind, M. A. (1997). Effects of Alzheimer's disease severity on cerebrospinal fluid norepinephrine concentration. Am. J. Psychiatry 154, 25-30. doi: 10.1176/ajp.154.1.25

Ferretti, M. T., Iulita, M. F., Cavedo, E., Chiesa, P. A., Schumacher, D. A., and Santuccione, C. A. (2018). Sex differences in Alzheimer disease - the gateway to precision medicine. Nat. Rev. Neurol. 14, 457-469. doi: 10.1038/s41582-018-0032-9

Fitzgerald, P. J. (2010). Is elevated norepinephrine an etiological factor in some cases of Alzheimer's disease? Curr. Alzheimer Res. 7:506. doi: $10.2174 / 156720510792231775$

Fliessbach, K., and Schneider, A. (2018). Biomarker-based diagnostics of Alzheimer's disease: concept of suspected non-Alzheimer pathology. Nervenarzt 89, 345-358. doi: 10.1007/s00115-018-0488-2

Giacobini, E., and Pepeu, G. (2018). Sex and gender differences in the brain cholinergic system and in the response to therapy of Alzheimer disease with cholinesterase inhibitors. Curr. Alzheimer Res. 15, 1077-1084. doi: 10.2174/1567205015666180613111504

Giunta, S., Ronchi, P., Valli, B., Franceschi, C., and Galeazzi, L. (2000). Transformation of beta-amyloid (A beta) (1-42) tyrosine to L-dopa as the result of in vitro hydroxyl radical attack. Amyloid 7, 189-193. doi: 10.3109/13506120009146833

Gordon, B. A., Blazey, T., Su, Y., Fagan, A. M., Holtzman, D. M., and Morris, J. C. (2016). Longitudinal beta-amyloid deposition and hippocampal volume in preclinical Alzheimer disease and suspected non-Alzheimer disease pathophysiology. JAMA Neurol. 73, 1192-1200. doi: 10.1001/jamaneurol.2016.2642

Griffiths, K. R., Leikauf, J. E., Tsang, T. W., Clarke, S., Hermens, D. F., and Efron, D. (2018). Response inhibition and emotional cognition improved by atomoxetine in children and adolescents with ADHD: the ACTION randomized controlled trial. J. Psychiatr Res. 102, 57-64. doi: 10.1016/j.jpsychires.2018.03.009

Grouzmann, E., and Lamine, F. (2013). Determination of catecholamines in plasma and urine. Best Pract. Res. Clin. Endocrinol. Metab. 27, 713-723. doi: 10.1016/j.beem.2013.06.004

Haywood, S. A., Simonian, S. X., van der Beek, E. M., Bicknell, R. J., and Herbison, A. E. (1999). Fluctuating estrogen and progesterone receptor expression in brainstem norepinephrine neurons through the rat estrous cycle. Endocrinology 140, 3255-3263. doi: 10.1210/endo.140.7.6869

Higgins, J. P., Thompson, S. G., Deeks, J. J., and Altman, D. G. (2003). Measuring inconsistency in meta-analyses. BMJ 327, 557-560. doi: $10.1136 /$ bmj.327.7414.557 
Higgins, J. P., Thompson, S. G., and Spiegelhalter, D. J. (2009). A re-evaluation of random-effects meta-analysis. J. R. Stat. Soc. Ser. A Stat. Soc. 172, 137-159. doi: 10.1111/j.1467-985X.2008.00552.x

Hows, M. E., Lacroix, L., Heidbreder, C., Organ, A. J., and Shah, A. J. (2004). High-performance liquid chromatography/tandem mass spectrometric assay for the simultaneous measurement of dopamine, norepinephrine, 5hydroxytryptamine and cocaine in biological samples. J. Neurosci. Methods 138, 123-132. doi: 10.1016/j.jneumeth.2004.03.021

Jaskiw, G. E., Kirkbride, B., and Bongiovanni, R. (2006). In rats chronically treated with clozapine, tyrosine depletion attenuates the clozapine-induced in vivo increase in prefrontal cortex dopamine and norepinephrine levels. Psychopharmacology (Berl) 185, 416-422. doi: 10.1007/s00213-005-0283-1

Kalinin, S., Polak, P. E., Lin, S. X., Sakharkar, A. J., Pandey, S. C., and Feinstein, D. L. (2012). The noradrenaline precursor L-DOPS reduces pathology in a mouse model of Alzheimer's disease. Neurobiol. Aging 33, 1651-1663. doi: 10.1016/j.neurobiolaging.2011.04.012

Kennedy, B. P., Bottiglieri, T., Arning, E., Ziegler, M. G., Hansen, L. A., and Masliah, E. (2004). Elevated S-adenosylhomocysteine in Alzheimer brain: influence on methyltransferases and cognitive function. J. Neural. Transm. (Vienna) 111, 547-567. doi: 10.1007/s00702-003-0096-5

Krashia, P., Martini, A., Nobili, A., Aversa, D., D’Amelio, M., and Berretta, N. (2017). On the properties of identified dopaminergic neurons in the mouse substantia nigra and ventral tegmental area. Eur. J. Neurosci. 45, 92-105. doi: 10.1111/ejn.13364

Kumari, S., Mukherjee, A., and Mukhopadhyay, C. K. (2018). Dopamine promotes cathepsin B-mediated amyloid precursor protein degradation by reactive oxygen species-sensitive mechanism in neuronal cell. Mol. Cell Biochem 454, 153-163. doi: 10.1007/s11010-018-3460-3

Kurup, R. K., and Kurup, P. A. (2003). Hypothalamic digoxin, hemispheric chemical dominance, and Alzheimer's disease. Intern. J. Neurosci. 113, 361-381. doi: 10.1080/00207450390162146

Lampe, T. H., Veith, R. C., Plymate, S. R., Risse, S. C., Kopeikin, H., and Cubberley, L. (1989). Pressor, norepinephrine, and pituitary responses to two TRH doses in Alzheimer's disease and normal older men. Psychoneuroendocrinology 14, 311-320. doi: 10.1016/0306-4530(89)90033-4

Liao, R. M., Lai, W. S., and Lin, J. Y. (2002). The role of catecholamines in retention performance of a partially baited radial eight-arm maze for rats. Chin. J. Physiol. 45, 177-185. doi: 10.1007/s10254-003-0008-y

Liu, L., Li, Q., Li, N., Ling, J., Liu, R., and Wang, Y. (2011). Simultaneous determination of catecholamines and their metabolites related to Alzheimer's disease in human urine. J. Separation Sci. 34, 1198-1204. doi: 10.1002/jssc.201000799

Lodeiro, M., Puerta, E., Ismail, M. A., Rodriguez-Rodriguez, P., Ronnback, A., and Codita, A. (2017). Aggregation of the inflammatory S100A8 precedes abeta plaque formation in transgenic APP mice: positive feedback for S100A8 and abeta productions. J. Gerontol A Biol. Sci. Med. Sci. 72, 319-328. doi: 10.1093/gerona/glw073

Luo, Y., Zhou, J., Li, M. X., Wu, P. F., Hu, Z. L., and Ni, L. (2015). Reversal of aging-related emotional memory deficits by norepinephrine via regulating the stability of surface AMPA receptors. Aging Cell 14, 170-179. doi: $10.1111 /$ acel.12282

Martinez-Morales, J. R., Morales, A., Marin, R., Hernandez-Jimenez, J. G., Acevedo, A., and Guerra, B. (2001). Estrogen modulates norepinephrineinduced accumulation of adenosine cyclic monophosphate in a subpopulation of immortalized luteinizing hormone-releasing hormone secreting neurons from the mouse hypothalamus. Neurosci. Lett. 298, 61-64. doi: 10.1016/S0304-3940(00)01722-5

Martorana, A., Di Lorenzo, F., Esposito, Z., Lo, G. T., Bernardi, G., and Caltagirone, C. (2013). Dopamine D(2)-agonist rotigotine effects on cortical excitability and central cholinergic transmission in Alzheimer's disease patients. Neuropharmacology 64, 108-113. doi: 10.1016/j.neuropharm.2012. 07.015

Moher, D., Liberati, A., Tetzlaff, J., and Altman, D. G. (2009). Preferred reporting items for systematic reviews and meta-analyses: the PRISMA statement. $B M J$ 339:b2535. doi: 10.1136/bmj.b2535

Mohsenzadegan, M., and Mirshafiey, A. (2012). The immunopathogenic role of reactive oxygen species in Alzheimer disease. Iran J. Allergy Asthma Immunol. $11,203-216$
Mosconi, L., Berti, V., Quinn, C., McHugh, P., Petrongolo, G., and Varsavsky, I. (2017). Sex differences in Alzheimer risk: brain imaging of endocrine vs chronologic aging. Neurology 89, 1382-1390. doi: 10.1212/WNL.0000000000004425

Nelson, A. J., Cooper, M. T., Thur, K. E., Marsden, C. A., and Cassaday, H. J. (2011). The effect of catecholaminergic depletion within the prelimbic and infralimbic medial prefrontal cortex on recognition memory for recency, location, and objects. Behav. Neurosci. 125, 396-403. doi: 10.1037/a0023337

Ounissi, M., Benkirane, A., Dempsey, E., Soares, R., Jullien, V., and Pons, G. (2015). A review of potential pharmacogenetic effects on catecholamine responses. Drug Metab. Rev. 47, 558-564. doi: 10.3109/03602532.2015.1102932

Pan, X., Kaminga, A. C., Wen, S. W., Wu, X., Acheampong, K., and Liu, A. (2019). Dopamine and dopamine receptors in Alzheimer's disease: a systematic review and network meta-analysis. Front. Aging Neurosci. 11:175. doi: 10.3389/fnagi.2019.00175

Pan, X., Wang, Z., Wu, X., Wen, S. W., and Liu, A. (2018). Salivary cortisol in post-traumatic stress disorder: a systematic review and meta-analysis. $B M C$ Psychiatry 18:9. doi: 10.1186/s12888-018-1910-9

Papp, K. V., Rentz, D. M., Mormino, E. C., Schultz, A. P., Amariglio, R. E., and Quiroz, Y. (2017). Cued memory decline in biomarkerdefined preclinical Alzheimer disease. Neurology 88, 1431-1438. doi: 10.1212/WNL.0000000000003812

Peaston, R. T., and Weinkove, C. (2004). Measurement of catecholamines and their metabolites. Ann. Clin. Biochem. 41, 17-38. doi: 10.1258/000456304322664663

Peskind, E. R., Elrod, R., Dobie, D. J., Pascualy, M., Petrie, E., and Jensen, C. (1998). Cerebrospinal fluid epinephrine in Alzheimer's disease and normal aging. Neuropsychopharmacology 19, 465-471. doi: 10.1016/S0893-133X(98)00054-2

Peskind, E. R., Wingerson, D., Murray, S., Pascualy, M., Dobie, D. J., and Le Corre, P. (1995). Effects of Alzheimer's disease and normal aging on cerebrospinal fluid norepinephrine responses to yohimbine and clonidine. Archives General Psychiatry 52, 774-782. doi: 10.1001/archpsyc.1995.03950210068012

Rajkumar, R., Kumar, J. R., and Dawe, G. S. (2017). Priming locus coeruleus noradrenergic modulation of medial perforant path-dentate gyrus synaptic plasticity. Neurobiol. Learn. Mem. 138, 215-225. doi: 10.1016/j.nlm.2016.07.003

Raum, W. J., and Swerdloff, R. S. (1981). A radioimmunoassay for epinephrine and norepinephrine in tissues and plasma. Life Sci. 28, 2819-2827. doi: 10.1016/0024-3205(81)90097-7

Rieckmann, A., Johnson, K. A., Sperling, R. A., Buckner, R. L., and Hedden, T. (2018). Dedifferentiation of caudate functional connectivity and striatal dopamine transporter density predict memory change in normal aging. Proc. Natl. Acad. Sci. U.S.A. 115, 10160-10165. doi: 10.1073/pnas.1804641115

Saldana, M., Pujols, L., Roca-Ferrer, J., Cardozo, A., Aguilar, E., and Bonastre, M. (2008). Relevance of COX-2 gene expression in dementia with lewy bodies associated with Alzheimer pathology. Mov. Disord 23, 804-810. doi: $10.1002 / \mathrm{mds} .21860$

Saxena, A. R., Chamarthi, B., Williams, G. H., Hopkins, P. N., and Seely, E. W. (2014). Predictors of plasma and urinary catecholamine levels in normotensive and hypertensive men and women. J. Hum. Hypertens 28, 292-297. doi: 10.1038/jhh.2013.112

Schraml, E., Quan, P., Stelzer, I., Fuchs, R., Skalicky, M., and Viidik, A. (2007). Norepinephrine treatment and aging lead to systemic and intracellular oxidative stress in rats. Exp. Gerontol. 42, 1072-1078. doi: 10.1016/j.exger.2007.08.003

Snowden, S. G., Ebshiana, A. A., Hye, A., Pletnikova, O., O’Brien, R., and Yang, A. (2019). Neurotransmitter imbalance in the brain and Alzheimer's disease pathology. J. Alzheimers Dis 72, 35-43. doi: 10.3233/JAD-190577

Soldan, A., Pettigrew, C., Cai, Q., Wang, M. C., Moghekar, A. R., and O'Brien, R. J. (2016). Hypothetical preclinical Alzheimer disease groups and longitudinal cognitive change. JAMA Neurol. 73, 698-705. doi: $10.1001 /$ jamaneurol.2016.0194

Stang, A. (2010). Critical evaluation of the Newcastle-Ottawa scale for the assessment of the quality of nonrandomized studies in meta-analyses. Eur. J. Epidemiol. 25, 603-605. doi: 10.1007/s10654-010-9491-z

Tank, A. W., and Lee, W. D. (2015). Peripheral and central effects of circulating catecholamines. Compr. Physiol. 5, 1-15. doi: 10.1002/cphy.c140007

Tatsumi, S., Uchihara, T., Aiba, I., Iwasaki, Y., Mimuro, M., and Takahashi, R. (2014). Ultrastructural differences in pretangles between Alzheimer disease and corticobasal degeneration revealed by comparative 
light and electron microscopy. Acta Neuropathol. Commun. 2:161. doi: 10.1186/s40478-014-0161-3

Umegaki, H., Ikari, H., Nakahata, H., Yoshimura, J., Endo, H., and Yamamoto, T. (2000). Low plasma epinephrine in elderly female subjects of dementia of Alzheimer type. Brain Res. 858, 67-70. doi: 10.1016/S0006-8993(99) 02440-3

Vermeiren, Y., Janssens, J., Aerts, T., Martin, J. J., Sieben, A., and Van Dam, D. (2016). Brain serotonergic and noradrenergic deficiencies in behavioral variant frontotemporal dementia compared to early-onset Alzheimer's disease. J. Alzheimers Dis. 53, 1079-1096. doi: 10.3233/JAD-160320

Walker, J. F., and Kane, C. J. (2002). Effects of body mass on nicotine-induced thermogenesis and catecholamine release in male smokers. Sheng Li Xue Bao $54,405-410$.

Yates, C. M., Simpson, J., and Gordon, A., Maloney, A. F., Allison, Y., and Ritchie, I. M. (1983). Catecholamines and cholinergic enzymes in pre-senile and senile Alzheimer-type dementia and down's syndrome. Brain Res. 280, 119-126. doi: 10.1016/0006-8993(83)91179-4

Youdim, M. (2018). Monoamine oxidase inhibitors, and iron chelators in depressive illness and neurodegenerative diseases. J. Neural. Transm. (Vienna) 125, 1719-1733. doi: 10.1007/s00702-018-1942-9
Zarow, C., Lyness, S. A., Mortimer, J. A., and Chui, H. C. (2003). Neuronal loss is greater in the locus coeruleus than nucleus basalis and substantia nigra in Alzheimer and Parkinson diseases. Arch. Neurol. 60, 337-341. doi: 10.1001/archneur.60.3.337

Zhang, Q., and Gong, M. (2016). On-line preconcentration of fluorescent derivatives of catecholamines in cerebrospinal fluid using flowgated capillary electrophoresis. J. Chromatogr. A 1450, 112-120. doi: 10.1016/j.chroma.2016.04.080

Conflict of Interest: The authors declare that the research was conducted in the absence of any commercial or financial relationships that could be construed as a potential conflict of interest.

Copyright $\odot 2020$ Pan, Kaminga, Jia, Wen, Acheampong and Liu. This is an openaccess article distributed under the terms of the Creative Commons Attribution License (CC BY). The use, distribution or reproduction in other forums is permitted, provided the original author(s) and the copyright owner(s) are credited and that the original publication in this journal is cited, in accordance with accepted academic practice. No use, distribution or reproduction is permitted which does not comply with these terms. 